\title{
Status of Measurement of $R$ value at BESIII
}

\section{Muzaffar Irshad ${ }^{a, *}$}

${ }^{a}$ Modern Physics Department, University of Science and Technology of China, 230026, Hefei, China

E-mail: subhani@mail.ustc.edu.cn

(On behalf of BESIII Collaboration)

In precision tests of the Standard Model, the observables such as the QED running coupling constant $\alpha\left(M_{Z}^{2}\right)$ evaluated at the $Z$ pole, and the anomalous magnetic moment $a_{\mu}^{\mathrm{SM}}$ of the muon, are of fundamental importance. The dominant uncertainties in both $\alpha\left(M_{Z}^{2}\right)$ and $a_{\mu}^{\mathrm{SM}}$ are due to the effects of hadronic vacuum polarization, which cannot be reliably calculated in the low energy region. This paper briefly reviewed the current status of measurement of $R$ values, using $e^{+} e^{-}$ collision data collected with BESIII detector at BEPCII storage ring in the center-of-mass energy range 2.2324 to $3.6710 \mathrm{GeV}$.

*** The European Physical Society Conference on High Energy Physics (EPS-HEP2021), ***

*** 26-30 July 2021 ***

*** Online conference, jointly organized by Universität Hamburg and the research center DESY ***

\footnotetext{
${ }^{*}$ Speaker
} 


\section{Introduction}

The lowest order cross section of $e^{+} e^{-} \rightarrow$ hadrons is usually parameterized in terms of $R$ value in units of the QED cross section for $e^{+} e^{-} \rightarrow \mu^{+} \mu^{-}$, namely $R=\sigma\left(e^{+} e^{-} \rightarrow\right.$ hadrons $) / \sigma\left(e^{+} e^{-} \rightarrow\right.$ $\left.\mu^{+} \mu^{-}\right)$, where $\sigma\left(e^{+} e^{-} \rightarrow \mu^{+} \mu^{-}\right)=\sigma_{\mu \mu}^{0}=4 \pi \alpha^{2} / 3 s$. This quantity plays crucial role in various precision tests of the Standard Model (SM) predictions, e.g., $R$ values at lower energies are employed to determine the hadronic contribution to the anomalous magnetic moment $a_{\mu}^{\mathrm{SM}}[1]$, and the value of the electromagnetic fine structure constant at the $Z$ pole $\alpha\left(M_{Z}^{2}\right)$, the running of the QED coupling constant $[2,3]$, which is essential to perform the precision physics at future colliders. As the dominating uncertainties in both $a_{\mu}^{\mathrm{SM}}$ and $\alpha\left(M_{Z}^{2}\right)$ are due to effect of imprecise calculation of determining the vacuum polarization at low energy region. $R$ value has been measured by many experiments especially at low energies below $5 \mathrm{GeV}$ [4-11], and most recent measurement with the KEDR detector has achieved a precision of better than $3.0 \%$ above $3.1 \mathrm{GeV}$ [11]. To reduce the error of $R$ value experimentally, is one of the primary goal at BESIII.

\section{Measurement of $\mathbf{R}$ value}

Experimentally, the $R$ value is determined by

$$
R=\frac{N_{\mathrm{had}}^{\mathrm{obs}}-N_{\mathrm{bkg}}}{\sigma_{\mu \mu}^{0} \mathcal{L}_{\mathrm{int}} \varepsilon_{\mathrm{trig}} \varepsilon_{\mathrm{had}}},
$$

where $N_{\text {had }}$ is the number of hadronic events directly counted from data, $N_{\mathrm{bkg}}$ is the remaining number of background events, $\mathcal{L}_{\text {int }}$ is the integrated luminosity of the data sample, $\varepsilon_{\text {trig }}$ is the trigger efficiency for hadronic events, $\varepsilon_{\text {had }}$ is the selection efficiency for generic hadronic events, and $(1+\delta)$ is the initial-state-radiation (ISR) correction factor, respectively.

\subsection{Event selection}

In this analysis, the hadronic events are selected in three steps. Potential sources backgrounds mainly comes from bhabha $\left(e^{+} e^{-} \rightarrow e^{+} e^{-}\right)$or di-gamma $\left(e^{+} e^{-} \rightarrow \gamma \gamma\right)$ processes due to their relatively large cross sections. In the first step, a large fraction of bhabha and di-gamma events are rejected by using the electromagnetic calorimeter (EMC) information only. In second step, the number of good charged hadronic tracks, which is referred to as prong hereafter, is selected by imposing the general track-based requirements at BESIII. In the last step, hadronic events are identified after implementing several further requirements. Fig. 1 shows the flow chart for the selection of hadronic events while measuring the $R$ value. It is noticed that the selected one-prong events are only used for the tuning of the signal MC model.

\subsection{Background events}

The residual background events mainly comes from $e^{+} e^{-} \rightarrow l^{+} l^{-}, \gamma \gamma$, and beam-associated processes. The remaining background events for $e^{+} e^{-} \rightarrow e^{+} e^{-}, \gamma \gamma$, and $\mu^{+} \mu^{-}$are estimated by the BABAYAGA 3.5 package [12], while that of $e^{+} e^{-} \rightarrow \tau^{+} \tau^{-}$events are estimated by KKMC [13]. The final state interaction among the particles and the BESIII detector simulation are described by GEANT4 [14]. The contribution of two-photon process $e^{+} e^{-} \rightarrow e^{+} e^{-} X$, with $X=e^{+} e^{-}, \mu^{+} \mu^{-}, \eta, \eta^{\prime}$, 


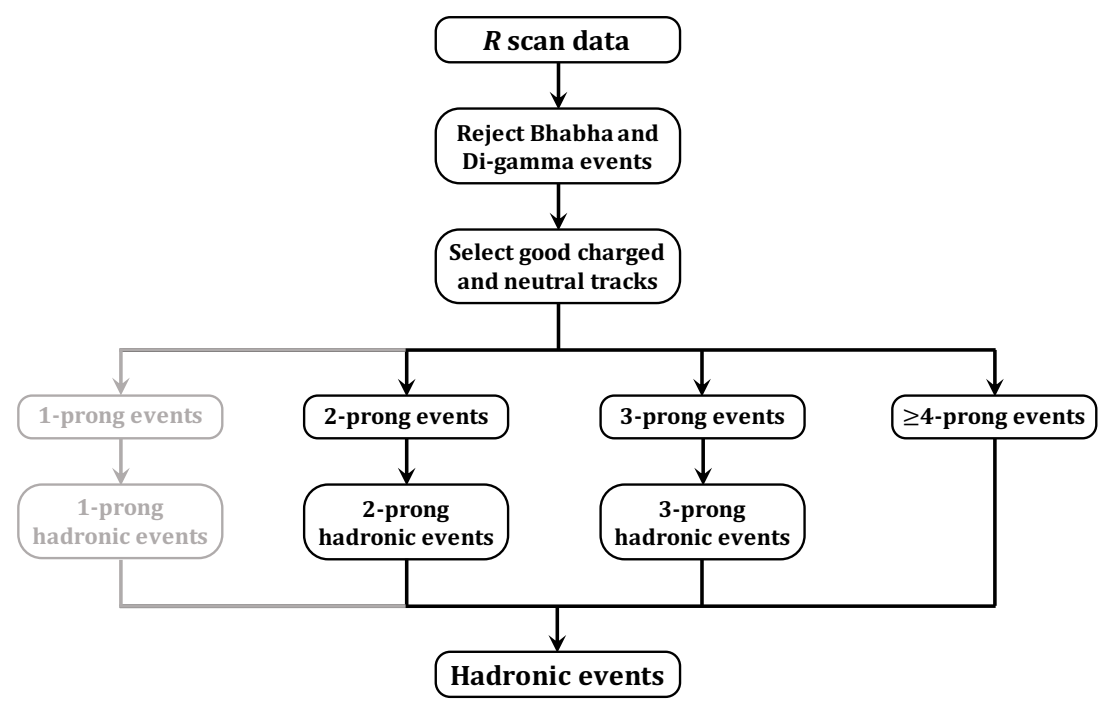

Figure 1: The flow chart of good hadronic events selection, where the prong means identified good charged track in each event.

$\pi^{+} \pi^{-}$and $K^{+} K^{-}[15]$ are considered by utilizing three dedicated MC generators [16]. The beamassociated background, which originates from beam-gas and beam-wall reactions, are non-collision backgrounds in hadronic events, and are estimated by sideband method with average vertex along the beam pipe direction.

\subsection{Hadronic event generator and ISR}

In the modern point of view, the hadronic production in the high energy electron-positron collision could be interpreted as processes illustrated in Fig. 2. The Lund area law generator LUARLW [17] is built in order to simulate the few-body production processes in BEPC energy region. LUARLW simulation contains following constitutes such as ISR, string fragmentation, multiplicity and momentum-energy distributions, decay of unstable hadrons.

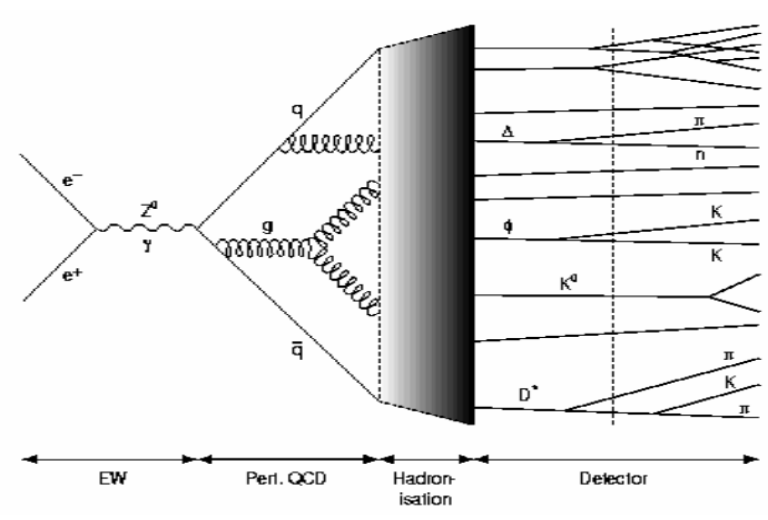

Figure 2: The hadronic production processes via virtual photon in $e^{+} e^{-}$annihilation. 
The Feynman diagram (FD) scheme is used to simulate the ISR process in the LUARLW generator and to calculate the correction factor $(1+\delta)$ after formalizing the contribution of the vacuum polarization (VP) correction with the complex expression $|1-\Pi(s)|^{2}$ instead of four real terms as presented in Ref. [18]. In addition, the contributions of leptons and narrow resonances to the VP operator $\Pi(s)$ are calculated analytically [18] and optical theorem [10].

\section{Sytematic uncertainty}

The major sources of systematic uncertainties originate from the simulation of the generic hadronic events and the calculation of the ISR correction. To estimate the corresponding uncertainty, an alternative generator has been developed, named as HYBRID. This generator integrates the CONEXC [20], PHOKHARA [21] and LuARLW models, where CONEXC able to simulates several exclusive process, PHOKHARA generates well parameterized and established exclusive channels, including $e^{+} e^{-} \rightarrow \pi^{+} \pi^{-}, \pi^{+} \pi^{-} \pi^{0}, \pi^{+} \pi^{-} \pi^{0} \pi^{0}$ and $2\left(\pi^{+} \pi^{-}\right)$, and remaining unknown decays are modeled by LUARLW [17], respectively. It is found that both LUARLW and HYBRID simulation models can described data well and result in consistent $R$ values. In HYBRID, the structure function scheme $[20,22]$ is used to simulate the ISR process and to calculate the corresponding correction factor. The difference of the calculated $(1+\delta)$ between the HYBRID and LUARLW simulations is found to be consistent within uncertainty.

\section{Summary and Outlook}

Using 14 electron-positron collision data samples collected with BESIII in the c.m.energies between 2.2324 to $3.6710 \mathrm{GeV}$, the $\mathrm{R}$ value measurement is in progress. The dominating uncertainties in the $R$ value calculations by the uncertainty of the hadron production model. The data analysis of $R$ value measurement has been essentially completed for below the open-charm energy region and will be published soon at BESIII. Further, the $R$ measurement at BESIII will be accomplish, and the whole $R$ program will be extended down to $2.0 \mathrm{GeV}$ and up to $4.9 \mathrm{GeV}$.

\section{Acknowledgment}

I am sincerely grateful to the EPS-HEP2021 committee for all of your hard work to provide such a well-organized and informative conference.

\section{References}

[1] T. Aoyama, N. Asmussen, M. Benayoun et al. Physics Reports 887, 1 (2020).

[2] M. Davier et al., Eur. Phys. J. C 71, 1515 (2011).

[3] K. Hagiwara et al., J. Phys. J. G 38, 085003 (2011).

[4] P. A. Rapidis et al., Phys. Rev. Lett. 39, 526 (1977).

[5] C. Bacci et al. ( $\gamma \gamma 2$ Collaboration), Phys. Lett. 86B, 234 (1979). 
[6] R. H. Schindler et al., Phys. Rev. D 21, 2716 (1980).

[7] J. L. Siegrist et al., Phys. Rev. D 26, 969 (1982).

[8] L. Criegee et al. (PLUTO Collaboration), Physics Reports 83, 151 (1982).

[9] J. Z. Bai et al. (BES Collaboration), Phys. Rev. Lett. 84, 594 (2000); J. Z. et al. (BES Collaboration), Phys. Rev. Lett. 88, 101802 (2002); M. Ablikim et al., (BES Collaboration), Phys. Lett. B 677, 239 (2009).

[10] V. V. Anashin et al. (KEDR Collaboration), Phys. Lett. B 753, 533 (2016); V. V. Anashin et al. (KEDR Collaboration), Phys. Lett. B 770, 174 (2017).

[11] V. V. Anashin et al. (KEDR Collaboration), Phys. Lett. B 788, 42 (2019).

[12] G. Balossini et al., Nucl. Phys. B 758, 227 (2006).

[13] S. Jadach, B. F. L. Ward and Z. Was, Comp. Phys. Commu. 130, 260 (2000); Phys. Rev. D. 63, 113009 (2001).

[14] S. Agostinelli et al. (Geant4 Collaboration), Nucl. Instrum. Meth. A 506, 250 (2003).

[15] V. M. Budnev et al., Physics Reports 15, 181 (1975).

[16] G. A. Schuler, Comput. Phys. Commun. 108, 279 (1998).

[17] B. Andersson, The Lund Model, Cambridge University Press, 1998; B. Andersson, and H. Hu, Few-body States in Lund String Fragmentation Model, arXiv:hep-ph/9910285.

[18] A. Osterheld et al., SLAC Report No. SLAC-PUB-4160 (1986).

[19] A. G. Shamov et al. (KEDR Collaboration), Chin. Phys. C 34, 836 (2010).

[20] H. Czyż, J. H. Kuhn, and S. Tracz, Phys. Rev. D 90, 114021(2014).

[21] R. G. Ping et al., Chin. Phys. C 40,113002 (2016).

[22] O. Nicrosini and L. Trentadue, Nucl. Phys. B 318, 1 (1989).

[23] F. Jegerlehner, Z. Phys. C 32, 195 (1986). 